\title{
Analyzing Energy Efficiency for IoT Devices with DRX Capability and Poisson Arrivals
}

\author{
Bowen Cai*, Yu Chen* and Izzat Darwazeh ${ }^{\dagger}$ \\ *National Engineering Lab for Mobile Network Technologies \\ *Beijing University of Posts and Telecommunications, Beijing, China \\ Email: \{cbw3011474, y.chen, cuiqimei\}@bupt.edu.cn \\ ${ }^{\dagger}$ Department of Electronic and Electrical Engineering, University College London (UCL), London, UK \\ Email: i.darwazeh@ucl.ac.uk
}

\begin{abstract}
Energy-efficient communications is one important consideration for Internet of Things (IoT) devices, and it can be achieved via the discontinuous reception (DRX) technology. In this paper, we consider an IoT device with the DRX capability. The device is functioning based on the LTE standard and it is communicating with the base station over a Nakagami- $m$ fading channel. Data are generated with fixed length and Poisson processes. Under these settings, we develop a cross-layer analytical model to analyze 1) the energy efficiency, 2) stationary probability and 3) state holding time of this device. Simulation results show that the proposed model can approximate the three performance of a IoT device accurately.
\end{abstract}

\section{INTRODUCTION}

The number of Internet of Things (IoT) devices grows rapidly and is expected to exceed the number of human user devices [1]. Most of the devices will be battery operated and require long life time with minimal maintenance. Therefore, it is important to save power consumption for better battery life. The discontinuous reception (DRX) is a core technology in mobile systems [2]; and It can dynamically switch off parts of the circuits from active state to short or long sleep state, resulting in significant energy savings.

Many researches have investigated DRX mechanisms under various traffic patterns or constraints to improve energy efficiency. Liang et al. [3] investigated a DRX optimization problem with the consideration of UEs' QoS requirements by maximizing UEs' sleep periods under a guaranteed bit rate. Feng et al. [4] introduced a playout buffer and proposed a DRX aware scheduling scheme to meet video streaming QoS requirements. Wang et al. [5] studied modeling and optimizing LTE DRX operations under self-similar traffic. Chu et al. [6] investigate the DRX mechanism in a batch server system, which allocates the resources according to the number of UEs, the number of backlog packets, quality of service (QoS) requirements and the wireless channel conditions of the UE. However, the above DRX models optimize DRX under the assumption that the channel quality is static. This assumption is in general not true for time-varying wireless fading channels.

An IoT device or a UE is in an active state if the device has data to transmit in its buffer. Therefore, we argue that the probability of a device being in a active state is associated with the non-empty buffer probability, when a wireless system is modeled as a queueing system. This probability over a wireless fading channel can be characterized by the effective capacity model [7] and was first derived in [8]. Based on the work of [8], Xu et al. [9], [10] considered a two-mode circuitry with an idle mode and an active mode, and further develop a two-mode energy efficiency analysis model. Their work considered the traffic model with a Bernoulli arrival process and exponentially distributed packet length. However, the data size for IoT applications is relatively fixed and their arrivals are better modeled as Poisson processes.

The aim of this paper is to find a way to analyze energy efficiency for IoT applications with DRX capability. In particular, we take LTE system as an example. Considering an uncorrelated Nakagami- $m$ fading channel, we model the IoT data packets as the Poisson arrival process with a fixed packet length. According to our proposed model, stationary probability and state holding time can be accurately calculated. Furthermore, energy efficiency can be analyzed and optimized by our proposed model.

The remainder of this paper is organized as follows. Section II describes the wireless DRX system model. In section III, we analyzed the stationary probability and energy efficiency of our proposed DRX model. Simulation results validate our proposed IoT energy efficiency model in Section IV, followed by a conclusion in Section V. The notation used in this paper is shown in table I.

\section{WIRELESS DRX SYSTEM MODEL}

\section{A. DRX mechanism}

The DRX mechanism in LTE is enabled in both the RRC_Idle and the RRC_Connected states. This is specified in the 3GPP standard TS 36.321 [11]. In this paper, we consider that the UE is always in the RRC_Connected state.

As shown in Fig.1, in RRC_Connected state, the whole data transmission process can be divided into four states, namely the active state, the DRX awake state, the short DRX state, and the long DRX state. In the active state, the UE receives data continuously from the base station and have a highest battery consumption. When there is no traffic arrival, the UE enters the DRX awake state and the Inactivity time $t_{i}$ begins. In the DRX awake state, the UE continuously monitors the PDCCH until the inactivity timer exceeds the limit value. Then the UE shuts down its transceiver circuitry and enters into short sleep 
TABLE I: Table I: Notation List

\begin{tabular}{l|l}
\hline Parameters & Values \\
\hline$p_{b}$ & nonempty buffer probability \\
\hline$S_{0}$ & active state \\
\hline$S_{1}$ & DRX awake state \\
\hline$S_{2}$ & short DRX state \\
\hline$S_{3}$ & long DRX state \\
\hline$\pi_{i}$ & stationary probability at $S_{i}$ \\
\hline$H_{i}$ & state holding time at $S_{i}$ \\
\hline$p_{i, j}$ & transition probability from $S_{i}$ to $S_{j}$ \\
\hline$t_{i}$ & Inactivity Timer \\
\hline$t_{s}$ & Short DRX Cycle Timer \\
\hline$t_{l}$ & Long DRX Cycle Timer \\
\hline$T$ & average system time \\
\hline$E$ & total energy consumption \\
\hline$P_{c}$ & circuit power \\
\hline$P_{t x}$ & transmission power \\
\hline$p_{t x}$ & transmission probability \\
\hline
\end{tabular}

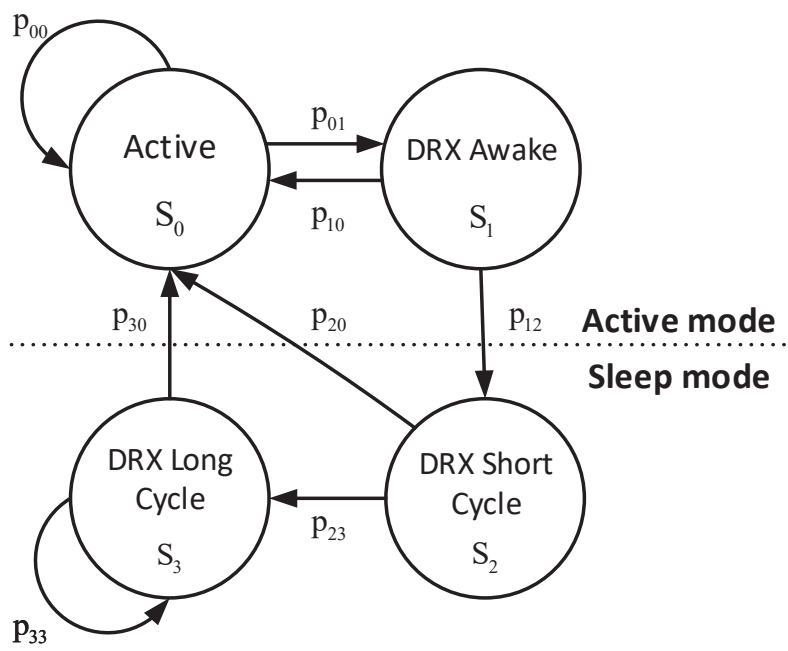

Fig. 1: State Transition

state with low power consumption. It wakes up and listens to $\mathrm{PDCCH}$ periodically in the short DRX state. If there is no packet received within the short DRX timer $t_{s}$ expires, which indicates the number of short DRX cycles has expired, the UE enters in the the long DRX state.

In the short DRX state and the long DRX state, the UE powers off transceiver circuitry and has lower energy consumption comparing to the UE in the active state and the DRX awake state.

\section{B. Traffic Model}

We consider an IoT device working in a block-fading wireless channel, as shown in Fig.2. The time slot of the device is $T_{s}$ seconds, which is equal to the fading time. Due to IoT devices typically have a small packet size but a fixed packet length, we assume that

1) the source arrival packet confirms to a Poisson distribution with parameter $\lambda$;

2) and the packet length is fixed with $L$ bits.

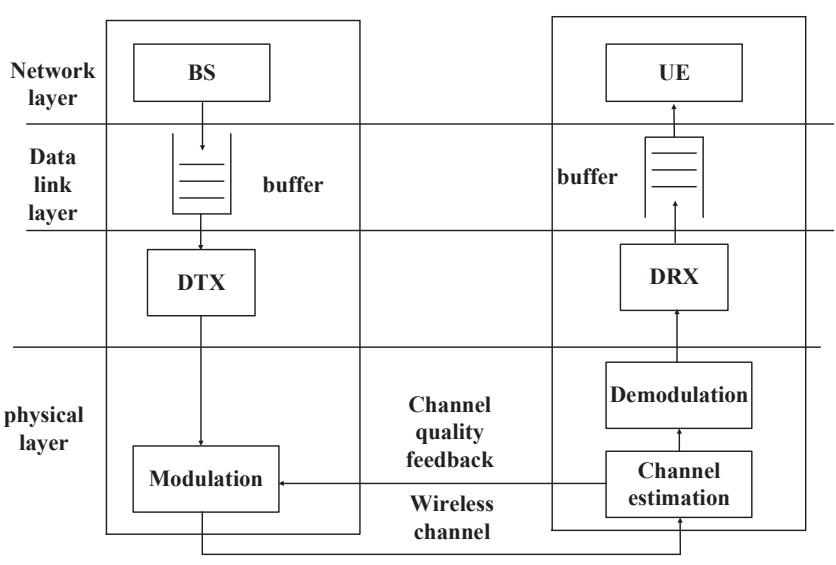

Fig. 2: Cross-layer system model

The data arrivals for the IoT device is denoted by $A[n]$ ( $n=$ $\{1,2,3, \ldots\})$, which is independent and identically distributed. Therefore, the probability of the $k(k=1,2, \ldots)$ packets arrival is

$$
P(A=k \text { packets per slot })=\frac{\lambda^{k}}{k !} e^{-\lambda} .
$$

Then the average arrival rate $\mu$ is

$$
\mu=\lambda L / T_{s} .
$$

The data arrival probability $p$ is

$$
p=1-P(A=0)=1-e^{-\lambda} .
$$

\section{Cross-Layer Analytical Model With DRX}

\section{A. Nonempty Buffer Probability Analysis}

Nakagami- $m$ block-fading channel extensively used for wireless channel modeling. The parameter $m$ is the shape parameter. When $m=1$, the signal experiences Rayleigh fading; when $m>1$, the signal experiences Rician fading. The probability distribution of signal-to-noise ratio (SNR) $\gamma$ in Nakagami- $m$ fading channel is [12]

$$
f_{\gamma}(\gamma)=\frac{\gamma^{m-1}}{\Gamma(m)}\left(\frac{m}{\bar{\gamma}}\right)^{m} \exp \left(-\frac{m}{\bar{\gamma}} \gamma\right)(\gamma \geq 0)
$$

where $\Gamma(\cdot)$ is the Gamma function, $\bar{\gamma}$ is the average SNR.

If the channel capacity $C[n]$ can be determined by Shannon's capacity, then the services $S[n]$ within a slot can be expressed as

$$
S[n]=T_{s} C[n]=T_{s} B_{c} \log _{2}(1+\gamma[n]),
$$

where $B_{c}$ is the channel bandwidth. Let $Q(n)$ denote the backlog size of the base station. Then

$$
Q(n+1)=\max (Q(n)+A(n)-S(n), 0)
$$

The complementary cumulative distribution function of backlog size $Q$ can be expressed as [7]

$$
P(Q>q) \approx p_{b} \exp \left(-u^{*} q\right) .
$$

where $p_{b}$ is the nonempty buffer probability and $u^{*}$ is a QoS exponent. 
From previous work such as [8] and [9], the QoS exponent can be derived by using effective bandwidth and effective capacity model. The QoS exponent $u^{*}$ satisfies the effective bandwidth $\alpha^{(b)}(u)$ equals to the effective capacity $\alpha^{(c)}(u)$, where

$$
\alpha^{(b)}(u)=\frac{\log (\mathrm{E}[\exp (u A)])}{T_{s} u}
$$

and

$$
\alpha^{(c)}(u)=-\frac{\log (\mathrm{E}[\exp (-u S)])}{T_{s} u} .
$$

As for the nonempty buffer probability in (7), it can be obtained by the following proposition.

Proposition 1: Consider a Poisson data arrival process with a fixed packet length and a Nakagami- $m$ fading channel, the nonempty buffer probability $p_{b}$ can be approximated by

$$
p_{b} \approx \frac{\sum_{k=0}^{+\infty} \int_{0}^{k L} \frac{\lambda^{k}}{k !} f_{S}(s) d s}{\sum_{k=0}^{+\infty} \int_{k L}^{+\infty} \frac{\lambda^{k}}{k !} e^{-(s-k L) u^{*}} f_{S}(s) d s}
$$

The proof is attached to the Appendix A.

\section{B. Stationary Probability and State Holding Time}

Denote $p_{i, j}$ and $\pi_{i}$ as the transition probability from $S_{i}$ to $S_{j}$ and the stationary probability of state $S_{i}$ respectively. The transition between states can happen in the following situations:

1) $S_{0}$ to $S_{1}$ happens when the buffer is empty and no traffic arrival at the base station;

2) $S_{1}$ to $S_{2}$ happens when the there is no traffic arrival until Inactivity timer $t_{i}$ expires;

3) $S_{2}$ to $S_{3}$ happens when there is no traffic arrival until short DRX timer $t_{s}$ expires;

4) $S_{i}(i=0,1,2,3)$ to $S_{0}$ happens when the UE monitor traffic arrival indicated by PDCCH in state $i$.

The transition between states can be modeled as the discreteparameter markov chains. Denote $\mathbf{P}$ as the state transition matrix, then $\mathbf{P}$ can expressed as

$$
\mathbf{P}=\left(\begin{array}{cccc}
p_{0,0} & p_{0,1} & 0 & 0 \\
p_{1,0} & 0 & p_{1,2} & 0 \\
p_{2,0} & 0 & 0 & p_{0,3} \\
p_{3,0} & 0 & 0 & p_{3,3}
\end{array}\right)
$$

For a DRX system, the data arrival at base station and buffer status are mutually independent, $p_{0,1}$ and $p_{0,0}$ can be expressed as

$$
\begin{aligned}
& p_{0,1}=(1-p)\left(1-p_{b}\right) \\
& p_{0,0}=1-(1-p)\left(1-p_{b}\right) .
\end{aligned}
$$

The other transition probabilities can be calculated as following

$$
\begin{aligned}
& p_{1,2}=(1-p)^{t_{i}} \\
& p_{1,0}=1-(1-p)^{t_{i}} \\
& p_{2,3}=(1-p)^{t_{s}} \\
& p_{2,0}=1-(1-p)^{t_{s}} \\
& p_{3,3}=(1-p)^{t_{l}} \\
& p_{3,0}=1-(1-p)^{t_{l}}
\end{aligned}
$$

Since the stationary vector $\pi=\left(\pi_{0}, \pi_{1}, \pi_{2}, \pi_{3}\right)$ satisfy $\pi \mathbf{P}=\pi$ and $\pi_{0}+\pi_{1}+\pi_{2}+\pi_{3}=1$. There exist a unique stationary distribution to ensure the autonomous DRX scheme has steady and consistent behavior. Based on the above notations for the statistical distributions, the stationary probabilities are

$$
\begin{aligned}
& \pi_{0}=\frac{p_{3,0}}{p_{3,0}\left(1+p_{0,1}+p_{0,1} p_{1,2}\right)+p_{0,1} p_{1,2} p_{2,3}} \\
& \pi_{1}=\frac{p_{0,1} p_{3,0}}{p_{3,0}\left(1+p_{0,1}+p_{0,1} p_{1,2}\right)+p_{0,1} p_{1,2} p_{2,3}} \\
& \pi_{2}=\frac{p_{0,1} p_{1,2} p_{3,0}}{p_{3,0}\left(1+p_{0,1}+p_{0,1} p_{1,2}\right)+p_{0,1} p_{1,2} p_{2,3}} \\
& \pi_{3}=\frac{p_{0,1} p_{1,2} p_{2,3}}{p_{3,0}\left(1+p_{0,1}+p_{0,1} p_{1,2}\right)+p_{0,1} p_{1,2} p_{2,3}}
\end{aligned}
$$

Since the total energy consumption depends on the time duration in each state, it is important to evaluate the holding time of different states. At $S_{0}$, the packet arrivals are the data stored in the buffer during the sleep cycle. We assume the UE can receive these data in $T_{s}$ seconds. This assumption is based on the fact that the peak rate of the LTE systems is more than $120 \mathrm{Mb} / \mathrm{s}$ [5]. Then $H_{0}$ is

$$
H_{0}=T_{s} .
$$

At $S_{1}$, the new packet arrives before the expiry of inactivity timer $t_{i}$. We assume $t_{i}$ expires at $K$ subframes (that is $t_{i}=$ $K T_{s}$ ), the probability that the time packet arrives at the $i$ th subframe is $p(1-p)^{i}$ and the holding time is $i T_{s}$ seconds, then the holding time at $S_{1}$ is

$$
H_{1}=\sum_{i=1}^{K} p(1-p)^{i} i T_{s} .
$$

The calculation of the $H_{2}$ and $H_{3}$ are similar to that of $H_{1}$. The main differences are we assume $t_{s}$ expires at $L$ subframes and $t_{l}$ expires at $M$ subframes respectively. Then the holding time at $S_{2}$ and $S_{3}$ are

$$
H_{2}=\sum_{i=1}^{L} p(1-p)^{i} i T_{s} .
$$

and

$$
H_{3}=\sum_{i=1}^{M} p(1-p)^{i} i T_{s} .
$$

\section{Energy Efficiency Analysis}

In a DRX system, the IoT device works either in the active mode with high energy consumption or in the sleep mode otherwise. In the active mode, the total power consumption $P_{\text {active }}$ includes circuit power consumption $P_{c}$ and transmission power consumption $P_{t x}$. In the sleep mode, the IoT device only have circuit power consumption. Then $P_{\text {active }}$ and $P_{\text {sleep }}$ are

$$
P_{\text {active }}=P_{c}+P_{t x}
$$

and

$$
P_{\text {sleep }}=P_{c} \text {. }
$$




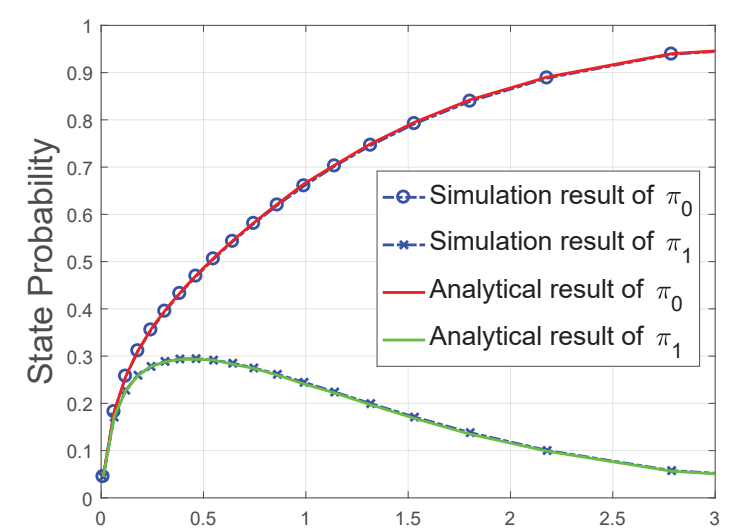

Average Arrival Rate, $\lambda$ (Packets per Second)

(a) $\pi_{0}$ and $\pi_{1}$

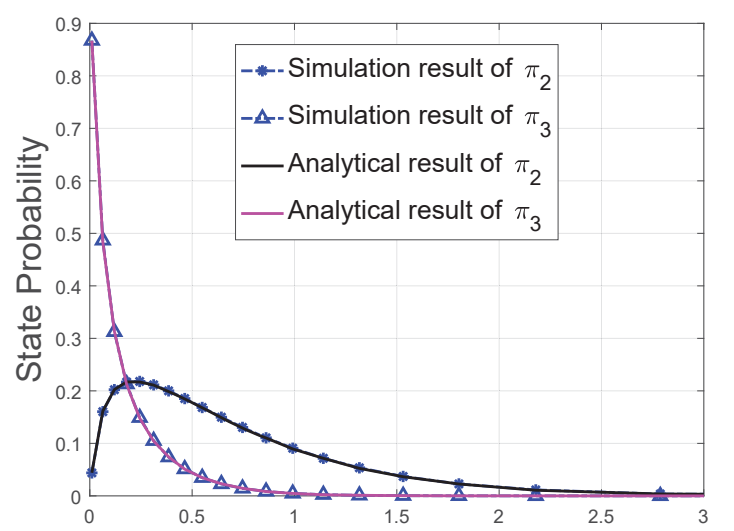

Average Arrival Rate, $\lambda$ (Packets per Second)

(b) $\pi_{2}$ and $\pi_{3}$

Fig. 3: State probability under different average arrival rate $\lambda$

Denote $T$ as the average system time, then $T$ can be expressed as

$$
T=\pi_{0} H_{0}+\pi_{1} H_{1}+\pi_{2} H_{2}+\pi_{3} H_{3} .
$$

In $S_{0}$ and $S_{1}$, the transmitter is in active mode and in $S_{2}$ and $S_{3}$, the transmitter is in sleep mode. Then the total energy consumption $E$ in average system time is

$$
\begin{aligned}
E & =\left(\pi_{0} H_{0}+\pi_{1} H_{1}\right)\left(P_{c}+P_{t x}\right)+\left(\pi_{2} H_{2}+\pi_{3} H_{3}\right) P_{c} \\
& =P_{c} T+\left(\pi_{0} H_{0}+\pi_{1} H_{1}\right) P_{t x} .
\end{aligned}
$$

Bits per Joule is an effective measurement to measure a system energy efficiency. For our proposed DRX system, the energy efficiency $\eta$ is the total power consumption $E$ in average system time

$$
\eta=\frac{\mu T}{E}=\frac{\mu T}{P_{c} T+\left(\pi_{0} H_{0}+\pi_{1} H_{1}\right) P_{t x}} .
$$

\section{Result And Discussion}

In this section, a simulation model is constructed to validate against the analytical model by using MATLAB as a simulation tool. For the result here, the parameters used in the simulations are $t_{i}=1 \mathrm{~ms}, t_{s}=3 \mathrm{~ms}, t_{l}=5 \mathrm{~ms}$ and $t_{i}=3 \mathrm{~ms}, t_{s}=5 \mathrm{~ms}$, $t_{l}=10 \mathrm{~ms}$ respectively. The other parameters are listed in Table II.

Table II: Simulation Parameters

\begin{tabular}{|c|l|}
\hline Parameter & Value \\
\hline Time duration of a slot, $T_{s}$ & $1 \mathrm{~ms}$ \\
\hline Packet length, $L$ & $500 \mathrm{bit}$ \\
\hline Spectral bandwidth, $B$ & $180 \mathrm{KHz}$ \\
\hline Average SNR, $\bar{\gamma}$ & $10 \mathrm{~dB}$ \\
\hline Data arrival probability, $p$ & $0.1,0.2, \ldots, 1.0$ \\
\hline active power consumption, $P_{t x}$ & $46 \mathrm{dBm}$ \\
\hline circuit power, $P_{c}$ & $0.1 \mathrm{~W}$ \\
\hline
\end{tabular}

Fig. 3 shows the state stationary probability under different $\lambda$. The simulation curves and analytical curves coincide with

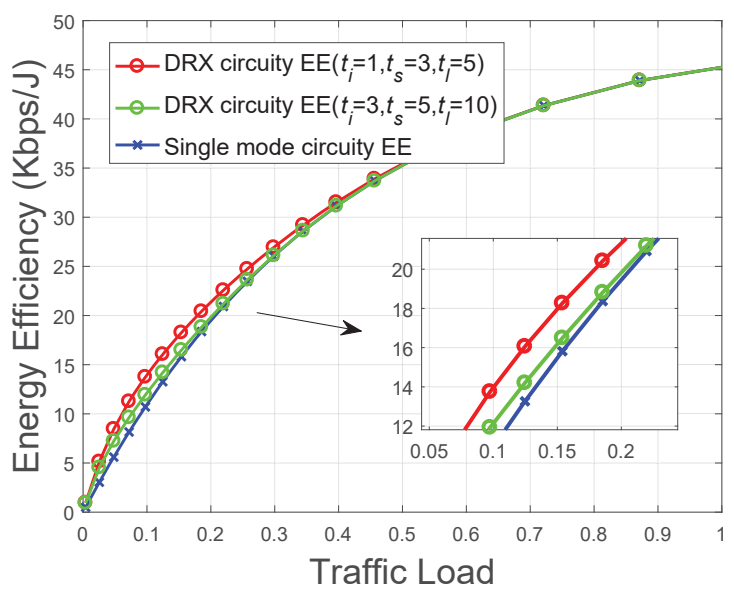

Fig. 4: Achieved energy efciency under different traffic load

each other, which verifies the accuracy of our proposed model. We can observe how the active state, DRX awake state, short DRX state, and long DRX state vary according to the different parameter $\lambda$. As $\lambda$ increases, the data arrival probability in (2) increases accordingly. From the figures, we can find $\pi_{0}$ is an increasing function of the data arrival probability, $\pi_{3}$ is a decreasing function of the data arrival probability. But $\pi_{1}$ and $\pi_{2}$ firstly increase and then decrease, so they have marked peak values at a certain data arrival rate.

Fig. 4 shows a comparison of our proposed DRX model energy efficiency $\left(t_{i}=1 \mathrm{~ms}, t_{s}=3 \mathrm{~ms}, t_{l}=5 \mathrm{~ms}\right.$ and $t_{i}=3 \mathrm{~ms}$, $\left.t_{s}=5 \mathrm{~ms}, t_{l}=10 \mathrm{~ms}\right)$ and single mode circuitry energy efficiency. From the figure, we can observe that the energy efficiency improvement compared with single mode circuitry. As the traffic load increases, the rate of growth decreases accordingly. The energy efficiency improvement is remarkable in low traffic load.

Fig.5 shows the energy efficiency improvement under different traffic load. This figure shows the energy efficiency 


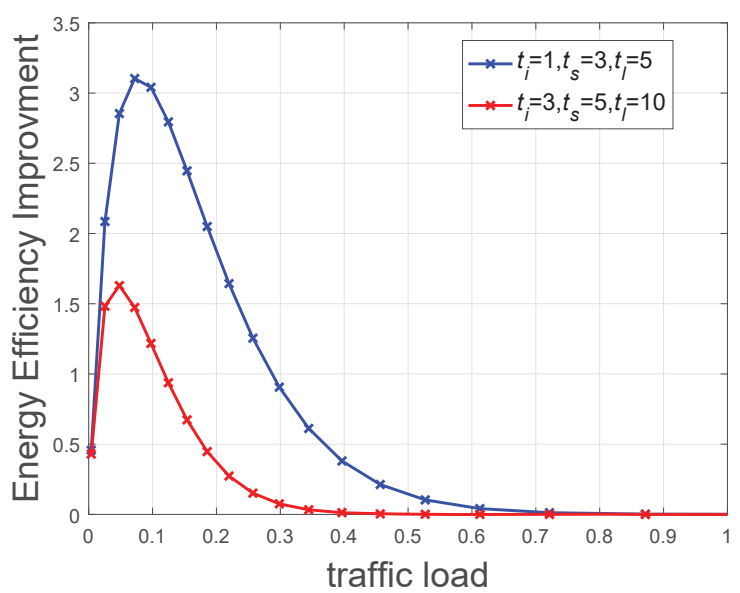

Fig. 5: Energy efciency improvement under different traffic load

improvement has a peak point at a certain traffic load. There exists an optimal traffic load that maximize the energy efficiency improvement.

\section{CONCLUSION}

It is important to understand the energy efficiency of Internet of Thing (IoT) devices. In this paper, a cross-layer analytical model is developed to analyze this property in LTE systems, and other two properties: stationary probability and state holding time. This model is based on the nonempty buffer probability of the effective capacity model and suitable for time-varying fading channels and data arrivals modeled as a Poisson process. Simulation results verify our analytical model and indicate the IoT device has a maximum energy efficiency improvement under a specific traffic load with DRX capability.

\section{ACKNOWLEDGEMENT}

This paper is supported by the National Science and Technology Major Project No. 2018ZX03001015-004.

\section{APPENDIX A}

When the data arrival process confirms the Poisson distribution and have a fixed packet length, the effective bandwidth $\alpha^{(b)}(u)$ can be expended as

$$
\alpha^{(b)}(u)=\frac{\log (\mathrm{E}[\exp (u A)])}{T_{s} u}=\frac{\lambda\left(e^{u L}-1\right)}{T_{s} u} .
$$

The effective capacity $\alpha^{(c)}(u)$ under Nakagami- $m$ fading channel is

$$
\alpha^{(c)}(u)=-\frac{1}{T_{s} u} \log \left(\int_{0}^{+\infty} \mathrm{e}^{-u T_{s} B_{c} \log _{2}(1+\gamma)} f_{\gamma}(\gamma) d \gamma\right) .
$$

Since the QoS exponent $u^{*}$ that satisfies:

$$
\begin{aligned}
& \alpha^{(b)}\left(u^{*}\right)=\alpha^{(c)}\left(u^{*}\right) \\
\Rightarrow \quad & E\left(e^{u^{*} A}\right) E\left(e^{-u^{*} S}\right)=1 \\
\Rightarrow & \sum_{k=0}^{+\infty} e^{k u^{*} L} \frac{\lambda^{k}}{k !} e^{-\lambda} \int_{0}^{+\infty} e^{-u^{*} s} f_{S}(s) d s=1,
\end{aligned}
$$

where $\Rightarrow$ is the implies symbol.

The nonempty buffer probability $p_{b}$ can be approximated by the method in [14]

$$
p_{b} \approx \frac{P(A>S)}{1-P\left(Q^{+}+A>S\right)+P(A>S)},
$$

where $Q^{+}$is the auxiliary variable and its probability density function $f_{Q^{+}}(b)$ is defined as

$$
f_{Q^{+}}(b)= \begin{cases}u^{*} e^{-u^{*} b} & (b \geq 0), \\ 0 & (b<0) .\end{cases}
$$

$P\left(Q^{+}+A>S\right)$ in (27) can be expended as

$$
\begin{aligned}
& P\left(Q^{+}+A>S\right)=P\left(Q^{+}+A>S \mid A \leq S\right)+P(A>S) \\
& =\sum_{k=0}^{+\infty} \int_{0}^{k L} P(A=k L) P\left(Q^{+}>s-k L\right) f_{S}(s) d s+P(A>S) \\
& =\sum_{k=0}^{+\infty} \int_{0}^{k L} \frac{\lambda^{k}}{k !} e^{-\lambda} e^{-(s-k L) u^{*}} f_{S}(s) d s+P(A>S) \\
& =1-\sum_{k=0}^{+\infty} \int_{k L}^{+\infty} \frac{\lambda^{k}}{k !} e^{-\lambda} e^{-(s-k L) u^{*}} f_{S}(s) d s+P(A>S)
\end{aligned}
$$

and $P(A>S)$ is

$$
\begin{array}{r}
P(A>S)=\int_{0}^{\infty} P(A>s) f_{S}(s) d s \\
=\sum_{k=0}^{+\infty} \int_{0}^{k L} \frac{\lambda^{k}}{k !} e^{-\lambda} f_{S}(s) d s .
\end{array}
$$

Therefore the nonempty buffer probability $p_{b}$ can be expressed by (26), (29) and (30)

$$
p_{b} \approx \frac{\sum_{k=0}^{+\infty} \int_{0}^{k L} \frac{\lambda^{k}}{k !} f_{S}(s) d s}{\sum_{k=0}^{+\infty} \int_{k L}^{+\infty} \frac{\lambda^{k}}{k !} e^{-(s-k L) u^{*}} f_{S}(s) d s}
$$

\section{REFERENCES}

[1] Recommendation ITU-R M.2083, "IMT Vision - 'Framework and overall objectives of the future development of IMT for 2020 and beyond," vol. ITU-R M.20, p. 21, 2015.

[2] TSGR, "TS 136213 - V14.4.0 - LTE; Evolved Universal Terrestrial Radio Access (E-UTRA); Physical layer procedures (3GPP TS 36.213 version 14.4.0 Release 14)," vol. 0, 2017.

[3] J. Liang and J. Chen, "An Energy-Efficient Sleep Scheduling With QoS Consideration in 3GPP LTE-Advanced Network for Internet of Things," IEEE Journal on Emerging and Selected Topics in Circuits and Systems, vol. 3, no. 1, pp. 13-22, March 2013.

[4] Y. Chen, G. Liu, and X. Chen, "Playout buffer aware scheduling scheme for video streaming over LTE networks," 2014 IEEE/CIC International Conference on Communications in China (ICCC), Shanghai, 2014, pp. 840-845.

[5] K. Wang, X. Li, H. Ji, and X. Du, "Modeling and Optimizing the LTE Discontinuous Reception Mechanism Under Self-Similar Traffic," IEEE Transactions on Vehicular Technology, vol. 65, no. 7, pp. 5595-5610, July 2016. 
[6] E. Chu, N.-O. Song, D. K. Sung, "A batch packet server (BPS) based discontinuous reception mechanism for energy saving," IEEE Transactions on Vehicular Technology, vol. 66, no. 11, pp. 10557-10561, Nov. 2017.

[7] D. Wu and R. Negi, "Effective capacity: A wireless link model for support of quality of service," IEEE Transactions on Wireless Communications, vol. 2, no. 4, pp. 630-643, July 2003.

[8] Y. Chen and I. Darwazeh, "An accurate approximation of delay with nakagami-m channels and exponential arrivals," 2015 IEEE Global Communications Conference (GLOBECOM), San Diego, CA, 2015, pp. 1-6.

[9] J. Xu, Y. Chen, Q. Cui, and X. Tao, "Use of Two-Mode Transceiver Circuitry and Its Cross-Layer Energy Efficiency Analysis," IEEE Communications Letters, vol. 21, no. 9, pp. 2065-2068, Sept. 2017.

[10] J. Xu, Y. Chen, H. Chen, Q. Cui, and X. Tao, "Use of Two-Mode Circuitry and Optimal Energy-Efficient Power Control Under Target DelayOutage Constraints," 2017 IEEE 28th Annual International Symposium on Personal, Indoor, and Mobile Radio Communications (PIMRC), Montreal, QC, 2017, pp. 1-7.

[11] Technical Specification Group Radio Access Network; Evolved Universal Terrestrial Radio Access (E-UTRA); Medium Access Control (MAC) Protocol Specification, 3GPP TS 36.321 V14.0.0, 2016

[12] W. Cheng, X. Zhang, and H. Zhang, "Statistical-QoS Driven EnergyEfficiency Optimization Over Green 5G Mobile Wireless Network," IEEE Journal on Selected Areas in Communications, vol. 34, no. 12, pp. 30923107, Dec. 2016.

[13] D. Wu and R. Negi, "Effective Capacity-Based Quality of Service Measures for Wireless," in First International Conference on Broadband Network, San Jose, CA, USA, 2004, pp. 527-536.

[14] Y. Chen and I. Darwazeh, "An Accurate Approximation of Delay with Nakagami-m Channels and Exponential Arrivals," in 2015 IEEE Global Communications Conference (GLOBECOM), San Diego, CA, 2015, pp. 1-6. 